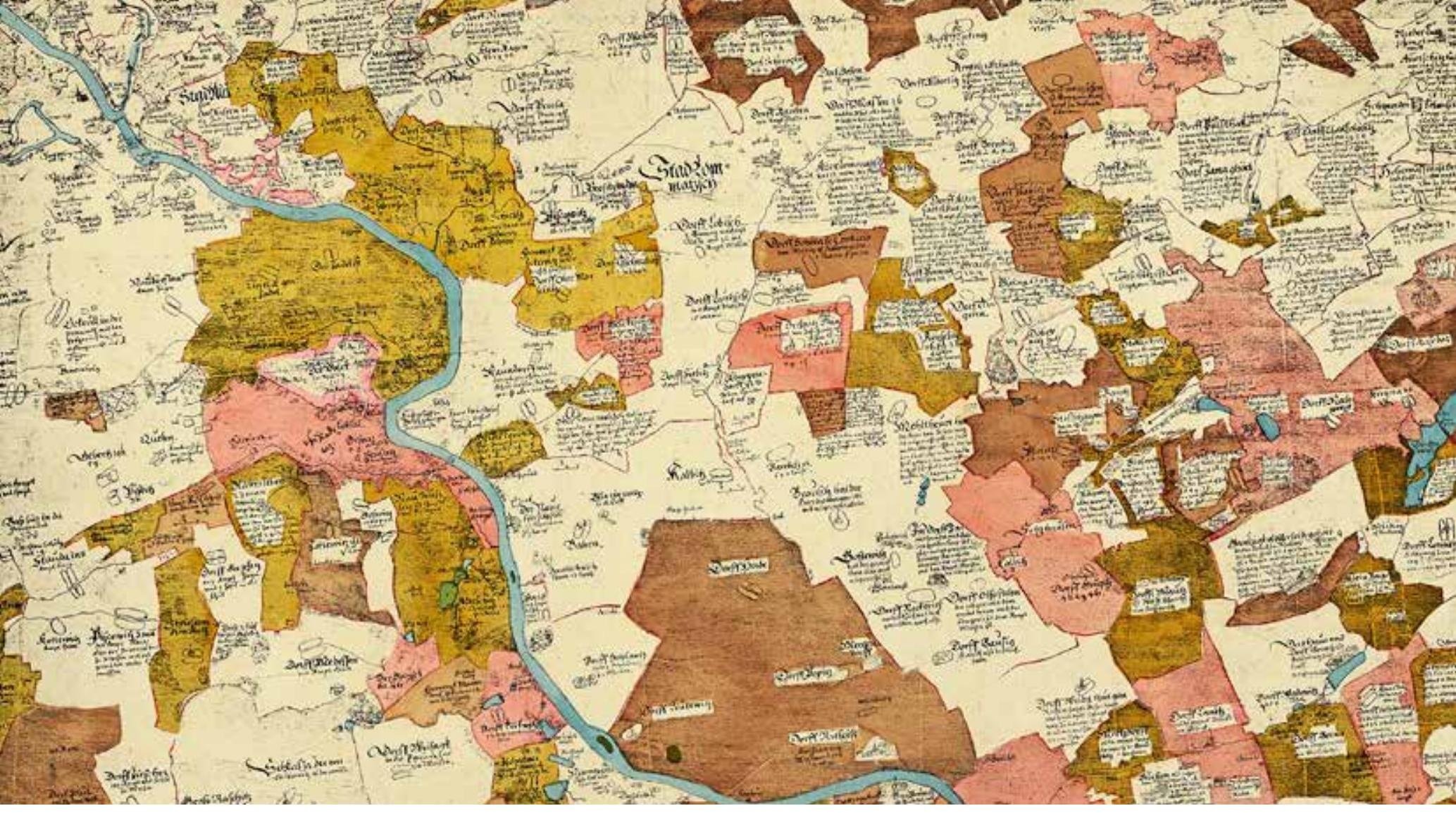

\title{
Zur Verwaltungsgeschichte der Lommatzscher Pflege
}

\author{
Matthias Donath
}

Die Lommatzscher Pflege ist eine sächsische Kulturlandschaft, die sich nicht klar umgrenzen lässt. Denn sie bildete nie eine geschlossene politische Einheit, weder als Herrschaft, als Amt oder als moderner Verwaltungsbezirk. Die Wahrnehmung dieses Raumes ist eng mit natürlichen Gegebenheiten verbunden, nämlich mit der erheblichen Bodenfruchtbarkeit und den hohen landwirtschaftlichen Erträgen. ${ }^{1}$ Schaut man sich eine Karte der Böden und Bodenwerte Sachsens an, dann liegt die Lommatzscher Pflege in jenem Landesteil, der sich durch enorm hohe Bodenwerte zwischen 80 und 100 Punkten auszeichnet und von anderen Landesteilen merklich abhebt. Sie ist identisch mit dem östlichen Teil des Mittelsächsischen Lößhügellandes. Dieser Naturraum ist durch Lößböden geprägt. Er besteht aus einem feinkörnigen Sediment, das sich in der Weichsel-Kaltzeit durch Windverfrachtung ablagerte und in seiner Zusammensetzung ideale Bedingungen für eine landwirtschaftliche Nutzung bietet.

Das, was wir heute unter „Lommatzscher Pflege“ verstehen, ist vor allem im 19. Jahrhundert niedergeschrieben worden, als die Landeskunde, die sich damals als Wissenschaft entwickelte, eine naturräumliche und geografische Gliederung Sachsens vornahm. Eine typische Zusammenfassung dessen, was wir als „Lommatzscher Pflege“ bis heute wahrnehmen, veröffentlichte Otto Eduard Schmidt (1855-1945) 1906 im dritten Band seiner „Kursächsischen Streifzüge“: „Das gesegnetste Stück des alten Meißner Landes ist ohne Zweifel die Lommatzscher Pflege. Bis auf den sagenumwobenen Bischof Benno wird der Ausspruch zurückgeführt, sie sei ,des Landes Myssen große Korntenne‘. Spätere bezeichneten sie als ,Sachsens Schmalzgrube; und noch heute [1906] heißen ihre Insassen im Volksmunde die ,Sammetbauern“."2 Die hier auftretenden Begriffe haben ältere Wurzeln. Philipp Melanchthon soll die Gegend um Döbeln bereits im 16. Jahrhunderts als „Sumen Misniae“ bezeichnet haben, als „Schmalztopf des Meißner Landes“, wie die Chronisten des 17. bis 19. Jahrhunderts mehrfach hervorhoben. ${ }^{3}$ Der Begriff „Sammetbauern“ bezieht sich darauf, dass die Besitzer der
Dörfer der Lommatzscher Pflege in der Kartierung durch Matthias Oeder, ausgeführt 1586 bis 1607 . Die Karte ist gesüdet; Norden ist unten.

๑ SLUB Dresden 
4 Neue Europäische Staatsund Reisegeographie worinnen die Lande des Obersächsischen Kreises ausführlich vorgestellet werden. Dresden/Leipzig 1755, S. 190191

5 Ebenda, S. 191.
Abschrift der Urkunde vom 1. September $1517 \mathrm{im}$ Chartularium des AugustinerChorherrenstifts St. Afra mit Erwähnung des Begriffes „Lommetzer pflege“" (grün unterstrichen) ๑ SLUB Dresden
Bauerngüter angeblich so wohlhabend waren, dass sie sich in Samt (Sammet) kleideten. Zunächst wurde dieser Begriff ausschließlich auf die Bauern des Altenburger Landes bezogen ${ }^{4}$ und erst später auf die Landschaft zwischen Döbeln und Meißen übertragen. Die Autoren der „Neuen Europäische Staats- und Reisegeographie“ sprachen 1755 von den „fetten Bauern“ „in dem so genannten Lomatzschen Grunde oder Pflege". 5 So wurde der Wohlstand der Landbewohner zum sprichwörtlichen Merkmal des Landschaftsraums. Der Volksmund sagte „De Lommatzscher Bauern tun's Geld mit
Scheffeln messen und de Butter mit Leffeln fressen.“

\section{Der Begriff „Lommatzscher Pflege“}

Interessanterweise ist die Lommatzscher Pflege, die ja keine definierten Verwaltungsgrenzen kennt und als kulturgeografischer Raum zu verstehen ist, mit einem Begriff gekennzeichnet, der ursprünglich aus der Verwaltungssprache kommt. Die Bezeichnung „Lommatzscher Pflege" erscheint erstmals in einer Urkunde des Augustiner-Chorherrenstifts St. Afra in Meißen

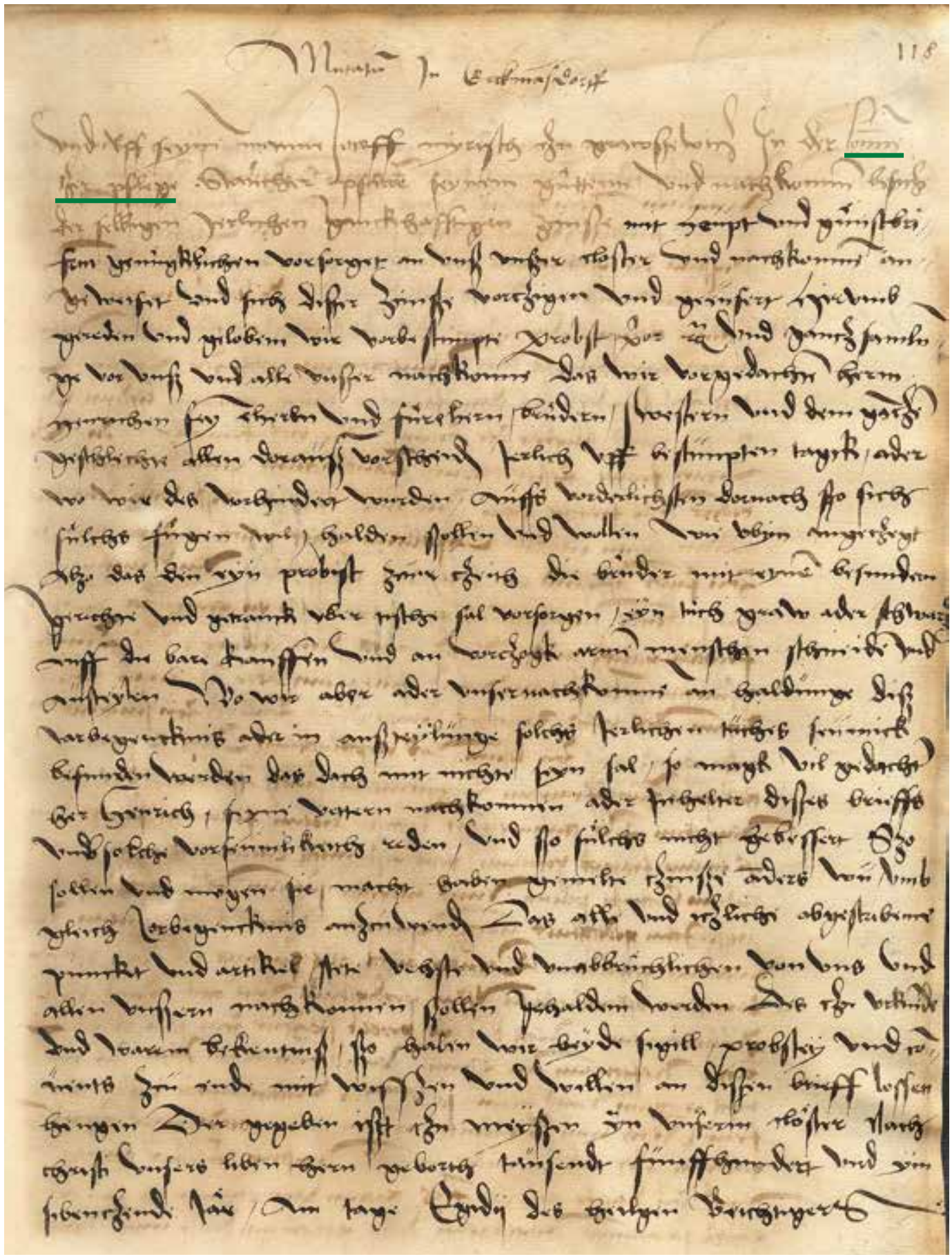




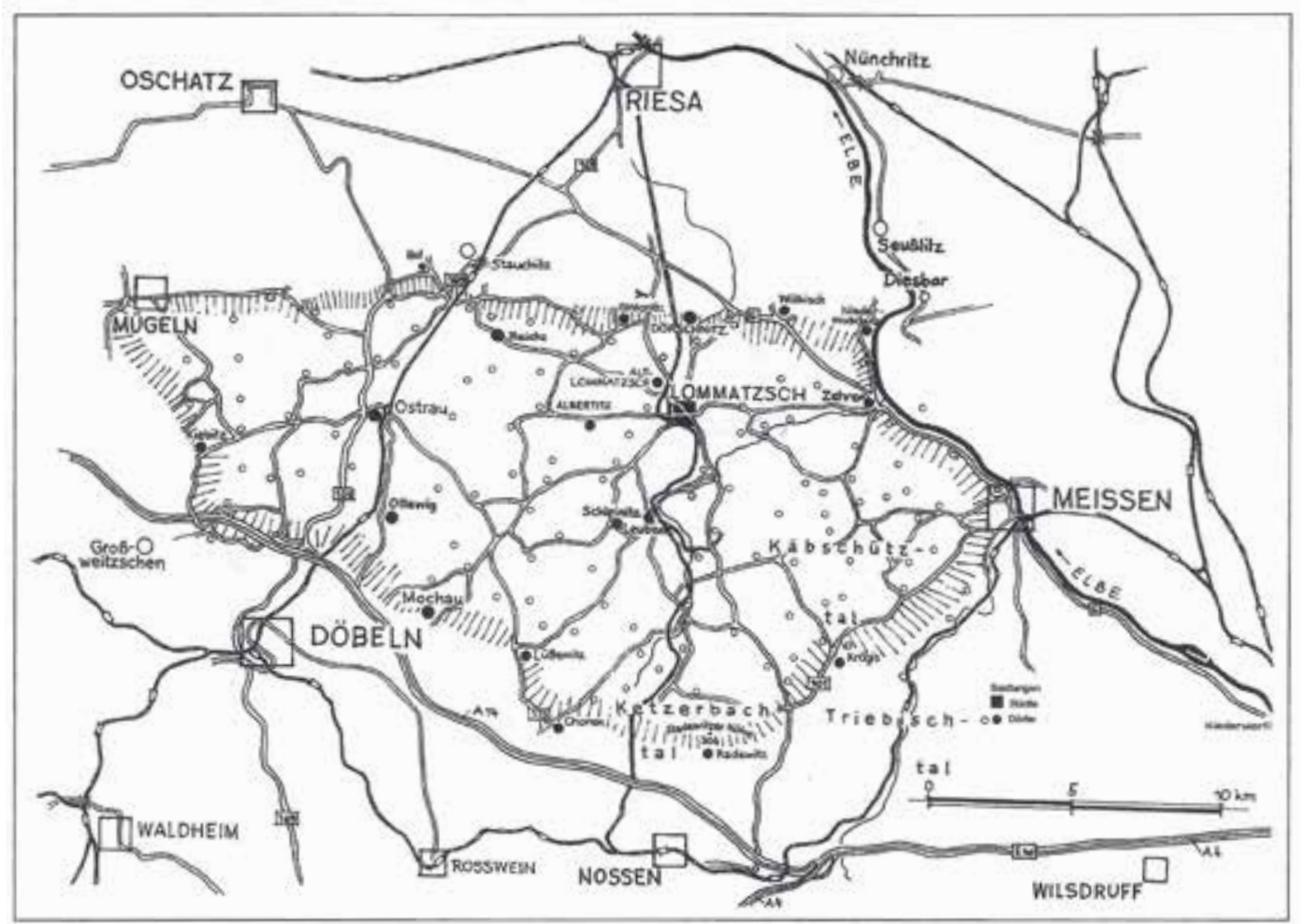

vom 1. September 1517. Damals bestätigte der Konvent die Stiftung des Domherrn Heinrich Mönch, Bruder des Rittergutsbesitzers Heinrich Mönch in Niederjahna, für ein Jahrgedächtnis in St. Afra. Dafür hatte „Jacoff Myrisch czu Grawßewicz in der Lommetzer pflege Staucher pfarre“ (Jakob Miersch aus Grauschwitz in der Lommatzscher Pflege, Pfarrei Staucha) jährliche Zinsen zu entrichten. ${ }^{6}$ Der Begriff „Pflege“ ist weitaus älter und schon vor 1517 gebraucht worden. Allerdings verband man ihn mit dem Stadtnamen Meißens. So listet eine Stiftungsurkunde des Burggrafen Heinrichs I. von Meißen vom 4. März 1409 für eine Kapelle des Stifts St. Afra Einkünfte aus mehreren Dörfern links der Elbe sowie aus „Lamaczsch“ auf. Zu diesen Orten wird gesagt: „alles in der pflege zcu Missin gelegin“.7 Der Landschaftsraum links der Elbe wurde somit zunächst nach der größten Stadt, Meißen, bezeichnet.

„Pflege“ bezeichnet einen Landbezirk, der von einem „Pfleger" verwaltet oder „gepflegt" wird. Dabei handelt es sich um die deutsche Entsprechung zu „Vogtei“ und „Amt“. Bereits im 14. Jahrhundert ist der Begriff „phlege“ im Herrschaftsgebiet der Wettiner bezeugt. ${ }^{8}$ Im 16. Jahrhundert wurde dieser ältere Begriff durch „Amt“ verdrängt. Wenn also 1409 von der „pflege zcu Missin“ die Rede war, dann meinte man das Amt Meißen, jenes Gebiet innerhalb der Mark Meißen, welches der Aufsicht des Amtmanns zu Meißen unterstand. Der Begriff „Pflege“ sagt noch nichts über die Boden- beschaffenheit oder die Ertragsstärke aus, sondern kennzeichnet ursprünglich lediglich einen Verwaltungsbezirk.

Erst im 16. Jahrhundert wurde der geografischpolitische Begriff mit der Kleinstadt Lommatzsch verbunden, ohne dass es jemals ein Amt Lommatzsch gegeben hätte. Damit bildete sich ein eigener Landschaftsbegriff heraus. Er setzte sich durch, während andernorts der Begriff „Pflege“ verschwand. Er wird in Sachsen sonst nur noch für die Großenhainer Pflege, die Kulturlandschaft um Großenhain rechts der Elbe, gebraucht. Dagegen haben sich andere, zeitweise in der Literatur nachweisbaren Bezeichnungen wie Mügelner oder Döbelner Pflege nicht durchgesetzt.

\section{Wechselnde Abgrenzungen}

Die Lommatzscher Pflege, so wie wir sie heute verstehen, war immer in mehrere politische Einheiten geteilt. Daher verfügt die Lommatzscher Pflege auch nicht über „historische“ Grenzen. So ist die Abgrenzung des zu behandelnden Gebietes äußerst schwierig. Viele Merkmale, die den Landschaftsraum kennzeichnen (der Lößboden, die vielen kleinen Dörfer), lassen sich nicht eindeutig abgrenzen.

Otto Eduard Schmidt beschrieb den Landschaftsraum wie folgt: „Die Lommatzscher Pflege erstreckt sich nordwärts gar nicht weit über das Städtchen hinaus, das ihr den Namen gegeben hat, nämlich nur bis zu der alten Leipzig-
Vorschlag einer Abgrenzung der Lommatzscher Pflege Zeichung: Dieter Bartusch
6 Ernst Gotthelf Gersdorf (Hrsg.): Urkundenbuch der Stadt Meißen und ihrer Klöster (Codex diplomaticus Saxoniae regiae II, 4), Leipzig 1873 (folgend CDS II, 4), Nr. 338.

7 Gersdorf 1873 (wie Anm. 6), Nr. 249, S. 200.

8 Christian Hesse: Amtsträger der Fürsten im spätmittelalterlichen Reich. Die Funktionseliten der lokalen Verwaltung in Bayern-Landshut, Hessen, Sachsen und Württemberg 1350-1515. Göttingen 2005, S. 120. 
9 Schmidt 1924 (wie Anm. 2), S. 73-74.

$10 \mathrm{Zu}$ den Ämtern vgl. Heinz Pannach: Das Amt Meißen vom Anfang des 14. bis zur Mitte des 16. Jahrhunderts. Studien zur Sozialstruktur, Verfassung und Verwaltung. Berlin 1960; Werner Stams: Die Ämter Meißen. In: Mitteilungen des Landesvereins Sächsischer Heimatschutz Heft 2/2009, S. 46-47; Karlheinz Blaschke/Uwe Ulrich Jäschke: Kursächsischer Ämteratlas 1790. Chemnitz 2009, S. 44-49.
Meißner Poststraße, die von Stauchitz in fast nördlicher Richtung an Dörschnitz vorüber bei Klappendorf in die neue Meißen-Oschatzer Straße einmündet. Ostwärts erreicht sie zwischen Niederlommatzsch und Zehren die Elbe, dann läuft die Grenze längs der Elbe bis nahe an Meißen heran, wendet sich mit der Nossener Straße südwärts und umfasst das beiderseits der Straße gelegene Land bis gegen die Höhe von Katzenberg hin und östlich bis zum Triebischtale hinab. Von Katzenberg geht die Grenze über Radewitz nach Choren und von da über Petersberg weit nach Nordwesten ausbiegend nach Mügeln und von da wieder ostwärts nach Stauchitz“.9 Der Verfasser der „Kursächsischen Streifzüge“ rechnete auch das Gebiet um Mügeln, südwestlich von Oschatz, zur Lommatzscher Pflege, das andere wiederum ausgliederten. Umstritten erscheint auch die Abgrenzung in der Umgebung Döbelns. Die Lommatzscher Pflege reicht mindestens bis an die Stadtgrenze Döbelns heran, doch kann auch ein Streifen westlich von Döbeln einbezogen werden.

Das gesamte Gebiet gehörte zur Mark Meißen, genauer: der ehemalige Gau Daleminzien war das eigentliche Herz der Mark Meißen. Demzufolge teilte die Lommatzscher Pflege alle politischen Entwicklungen, die das Herrschaftsgebiet der Markgrafen von Meißen sowie Herzöge, Kurfürsten und Könige von Sachsen betrafen. Unterhalb der Ebene der Landesherrschaft gab es aber Herrschafts- und Verwaltungsgrenzen. Diese blieben über Jahrhunderte weitgehend konstant. Erst die Reformen des 19. Jahrhunderts beendeten die herrschaftliche Zersplitterung.

\section{Vier Ämter Meißen}

Der größte Herrschaftskomplex innerhalb der Lommatzscher Pflege war das Amt Meißen, also jener landesherrliche Besitz, der von Meißen aus verwaltet wurde. ${ }^{10}$ Das Amt Meißen war die Mitte des 1547 gebildeten Meißner Kreises und der sächsischen Erblande und wurde daher oft auch „Kreisamt Meißen“ oder „Erbamt Meißen“ genannt. Nach der Einwohnerzahl war das Kreisamt Meißen eines der größten Ämter Kursachsens. Es wurde von den Ämtern Leisnig, Oschatz, Großenhain, Dresden, Grillenberg, Freiberg und Nossen begrenzt. Das Amtsgebiet reichte weit über die Lommatzscher Pflege hinaus, indem es etwa Dörfer um Wilsdruff und einige Orte rechts der Elbe umfasste. Die größte Stadt im Amtsgebiet war Meißen. Daneben gab es nur noch die Kleinstadt Lommatzsch. Ganz im Norden des Amtsgebiets hatte sich Riesa, ehemals ein Dorf, zu einem Marktflecken oder Städtchen entwickelt.
Das Kreisamt Meißen umfasste insgesamt 326 ländliche Siedlungen. Diese wiederum teilten sich in Amtsdörfer und Rittergutsdörfer. Der Anteil der Amtsdörfer war relativ gering. Nur 23 ganze Dörfer und 32 Dorfteile unterstanden unmittelbar dem Meißner Amtmann, der hier die grundherrlichen Rechte wahrnahm. Alle anderen Dörfer gehörten zu einem Rittergut. Die Grundherrschaften der Rittergüter waren unterschiedlich groß, sie konnten ein Dorf oder bis zu 16 Dörfer umfassen. Man unterschied zwischen amtssässigen und schriftsässigen Rittergütern. Die amtssässigen Rittergüter unterstanden dem Amt; die Rittergutsbesitzer wählten aus ihrer Mitte die Vertreter für die Ritterschaftskurie des sächsischen Landtags. Die Besitzer der schriftsässigen Rittergüter durften selbst an den Landtagssitzungen teilnehmen, sie erhielten landesherrliche Verfügungen nicht vom Amtmann, sondern von den Behörden in Dresden. 1792 gab es im Kreisamt Meißen 35 amtssässsige, 40 altschriftsässige und 13 neuschriftsässige Rittergüter.

Der Besitz geistlicher Einrichtungen unterlag nicht dem Zugriff des Kreisamts Meißen. Der größte kirchliche Grundeigentümer vor der Reformation war das Meißner Domkapitel gewesen. Es hatte den Grundbesitz seit dem 11. Jahrhundert durch zahlreiche Schenkungen und Stiftungen zusammengetragen. Vor der Reformation übte das Meißner Domkapitel die Grundherrschaft über rund 90 Dörfer und Dorfteile aus. Diese bildeten kein zusammenhängendes Gebiet, sondern lagen verstreut in der Mark Meißen zwischen Dresden und Strehla überwiegend links der Elbe. Dabei bildete die Lommatzscher Pflege den Kernraum des Kapitelsbesitzes. Die Einnahmen aus diesen Dörfern kamen bestimmten Personen (Domherren, Vikare) oder geistlichen Stiftungen zugute. Mit ihnen wurden etwa Seelenmessen oder Jahrgedächtnisse im Meißner Dom bezahlt.

Infolge der Reformation wurde das Meißner Domkapitel nicht aufgehoben. Es blieb als evangelische Einrichtung bestehen. Kurfürst August von Sachsen beließ dem Domkapitel aber nur einen kleinen Teil seines früheren Besitzes, und zwar jene Dörfer, die der Ausstattung der acht verbliebenen Domherrenstellen dienten. Etwa zwei Drittel des Grundbesitzes wurden 1565 einer gesonderten staatlichen Behörde unterstellt, dem Prokuraturamt Meißen, und damit dem Zugriff des Domkapitels entzogen. Die Behörde wurde von einem Prokuraturverwalter geleitet, der seinen Dienstsitz im Meißner Bischofsschloss hatte. Die Erträge der Prokuratur wurden für die Universität Leipzig, die Besoldung von Pfarrern und Lehrern sowie andere staatliche Aufgaben 
ausgegeben. Um Lommatzsch lagen mehrere Prokuraturdörfer: Jesseritz, Pröda, Priesa, Piskowitz, Prositz, Großkagen, Zöthain, Domselwitz, Pitschütz, Birmenitz, Lützschnitz, Beutig, Mischütz sowie Anteile von Berntitz und Wuhnitz.

Etwa 20 Dörfer und Dorfteile waren dem Meißner Domkapitel geblieben. Diese Stiftsdörfer waren im Stiftsamt Meißen zusammengefasst. Der Name verwirrt, denn die Dörfer gehörten nie zum Hochstift Meißen, dem staatsrechtlich eigenständigen Gebiet der Bischöfe von Meißen, das seit 1581 der weltlichen Hoheit der Kurfürsten von Sachsen unterstellt war. Die Verwaltung übte in diesen Dörfern nicht die Stiftsregierung in Wurzen aus, die das ehemals bischöfliche Stiftsgebiet regierte, sondern das Domkapitel in Meißen. Die Einnahmen aus den Dörfern speisten die Präbenden, jene Vermögensanteile, die jeweils einem bestimmten Domherren zugeordnet waren. Die größte Präbende war die Dompropstei. Ihr waren drei Dörfer in der Lommatzscher Pflege zugeordnet: Boritz, Mettelwitz, Prositz. Außerdem gehörten Abend, Lüttnitz, Noßlitz, Rüsseina, Sörnewitz, Wölkau, Zschaitz und Zschannewitz zu den Präbenden des Domkapitels. Weiter Dörfer unterstanden dem Dombaumeister, der die Einnahmen für den Unterhalt des Meißner Doms, aber auch für baufremde Ausgaben zu verwenden hatte. Die „Baumeisterei“ umfasste Saultitz, einen Anteil von Toppschädel und das Mühlengut Dreikutten, dazu vier Dörfer bei Wilsdruff.

Die zweitgrößte geistliche Einrichtung war das Augustiner-Chorherrenstift St. Afra. Auch dieses verfügte über Dörfer und Dorfanteile in der Lommatzscher Pflege. Nach der Auflösung des Stifts infolge der Reformation gelangte dieser Grundbesitz an die 1543 gestiftete Landesschule St. Afra. Diese erhielt 1568 zusätzlich Teile des Grundbesitzes des ebenfalls aufgelösten Nonnenklosters Heilig Kreuz bei Meißen. Aus den Besitzungen beider Klöster wurde das Schulamt Meißen gebildet, dessen Erträge für den Unterhalt der Meißner Fürstenschule bestimmt waren. Das Schulamt umfasste einen geografisch weit verteilten Streubesitz zwischen Meißen, Oschatz und Großenhain. Insgesamt waren es rund 40 Dörfer und Dorfteile. Schulamtsdöfer (oder kurz: Schuldörfer) innerhalb der Lommatzscher Pflege waren Oberlommatzsch, Naundorf, Gasern, Mohlis, Altlommatzsch und Gohris.

Kreisamt Meißen, Schulamt Meißen, Prokuraturamt Meißen und Stiftsamt Meißen wurden auch als „die vier Ämter Meißen“ zusammengefasst. Innerhalb dieser Ämter nahm Lommatzsch, der namengebende Ort der Lommatzscher Pflege, nie eine Verwaltungsfunktion ein. Verwaltungszentrum war ausschließlich Meißen.

\section{Einführung der Amtshauptmannschaften}

Zu den umfassenden Reformen, die im 19. Jahrhundert im Königreich Sachsen durchgeführt wurden, gehörte auch eine Verwaltungsreform. In den 1830er Jahren wurden die früheren Ämter abgeschafft und durch größere Amtshauptmannschaften ersetzt. 1874 wurde eine neue Verwaltungsgliederung vorgenommen und Sachsen in insgesamt 25 Amtshauptmannschaften geteilt. Dabei erfolgte eine Aufteilung jenes Raumes, der vorher in den vier Meißner Ämtern zusammengefasst war, und damit auch der Lommatzscher Pflege. Dabei versuchte man, die Dörfer den jeweils nächstgelegenen städtischen Zentren Meißen, Oschatz und Döbeln zuzuordnen. Lommatzsch selbst war zu klein, um Sitz einer Amtshauptmannschaft zu werden.

Die 1874 gebildete Amtshauptmannschaft Meißen war deutlich kleiner als das frühere Kreisamt Meißen. Ihr gehörten etwa zwei Drittel der Lommatzscher Pflege an, darunter auch Lommatzsch und Nossen. Der nördliche Teil der Lommatzscher Pflege, die Dörfer bei Riesa, wurde der Amtshauptmannschaft Großenhain zugeordnet. Dagegen gelangte der nordwestliche Teil, einschließlich Hof, an die Amtshauptmannschaft Oschatz. Der gesamte westliche Teil der Lommatzscher Pflege, darunter Zschaitz und Ostrau, wurde hingegen der Amtshauptmannschaft Döbeln angegliedert. Die Teilung der Lommatzscher Pflege wurde dadurch verstärkt, dass hier auch die Grenze der mittleren Verwaltungsbezirke verlief. Die Amtshauptmannschaften Döbeln und Oschatz gehörten der KreisamtmannschaftLeipzig, dieAmtshauptmannschaften Meißen und Großenhain der Kreishauptmannschaft Dresden an. Nach dieser Verwaltungsgliederung richteten sich auch die Kirchenbezirke (Ephorien) der Evangelisch-Lutherischen Landeskirche Sachsens. Sie wurden 1874 den Grenzen der Amtshauptmannschaften angepasst.

Die 1874 geschaffene Verwaltungsgliederung blieb bis in die frühen Jahre der DDR bestehen, nur mit dem Unterschied, dass 1938 neue Bezeichnungen eingeführt worden waren. Aus dem Amtshauptmannschaften waren nach preußischen Vorbild Landkreise und aus den Kreishauptmannschaften Regierungsbezirke geworden.

\section{Verwaltungsreformen}

Im Zusammenhang mit der Abschaffung der Länder in der DDR wurde 1952 eine Kreisreform durchgeführt. Diese wirkte sich insofern auf die Lommatzscher Pflege aus, weil sie die Aufteilung auf verschiedene Verwaltungseinheiten 
Verwaltungsgliederung der

Lommatzscher Pflege um 1790

Zeichenerklärung

Stadt über 10.000 Einwohner

๑ Stadt über 5.000 Einwohner

回 Stadt über 1.000 Einwohner

Stadt bis 1.000 Einwohner

○ Dorf (patrimonialer Grundherrschaft)

- Dorf (landesherrschaftlicher Grundherrschaft)

- Dorf (gemischte Grundherrschaft)

๑ Marktflecken

- Marktflecken

× Wüste Mark

$\uparrow \quad$ Pfarrkirche

$\dagger \quad$ Filialkirche

F landesherrliches Schloss

㔽L \& standesherrliches Schloss

P Kammergut

P $\quad$ Rittergut

\section{Grenze Kursachsen}

Amtsgrenzen

Stiftsamt Meißen

Schulamt Meißen

Kreisamt Meißen

Prokuraturamt Meißen

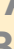
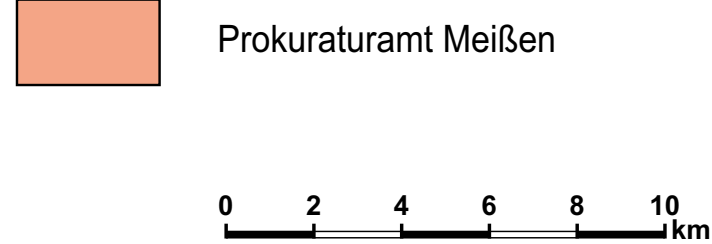

Zusammenstellung aus:

Blaschke, Karlheinz; Uwe Ulrich Jäschke: Kursächsischer Ämteratlas. Chemnitz 2008, S. 42-47.

Kartographie:

Uwe Ulrich Jäschke - Hochschule für Technik und Wirtschaft Dresden, Stand 2017.
Dahlen

A $m$ t

Strehla
O s c h a t z

e $\mathrm{n}$

茴Oschatz OWeida
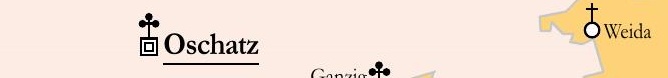

Amt Waldenbura
Ide

Amt
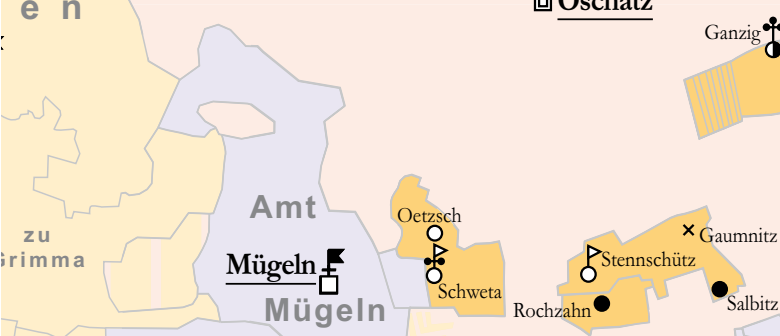

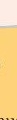




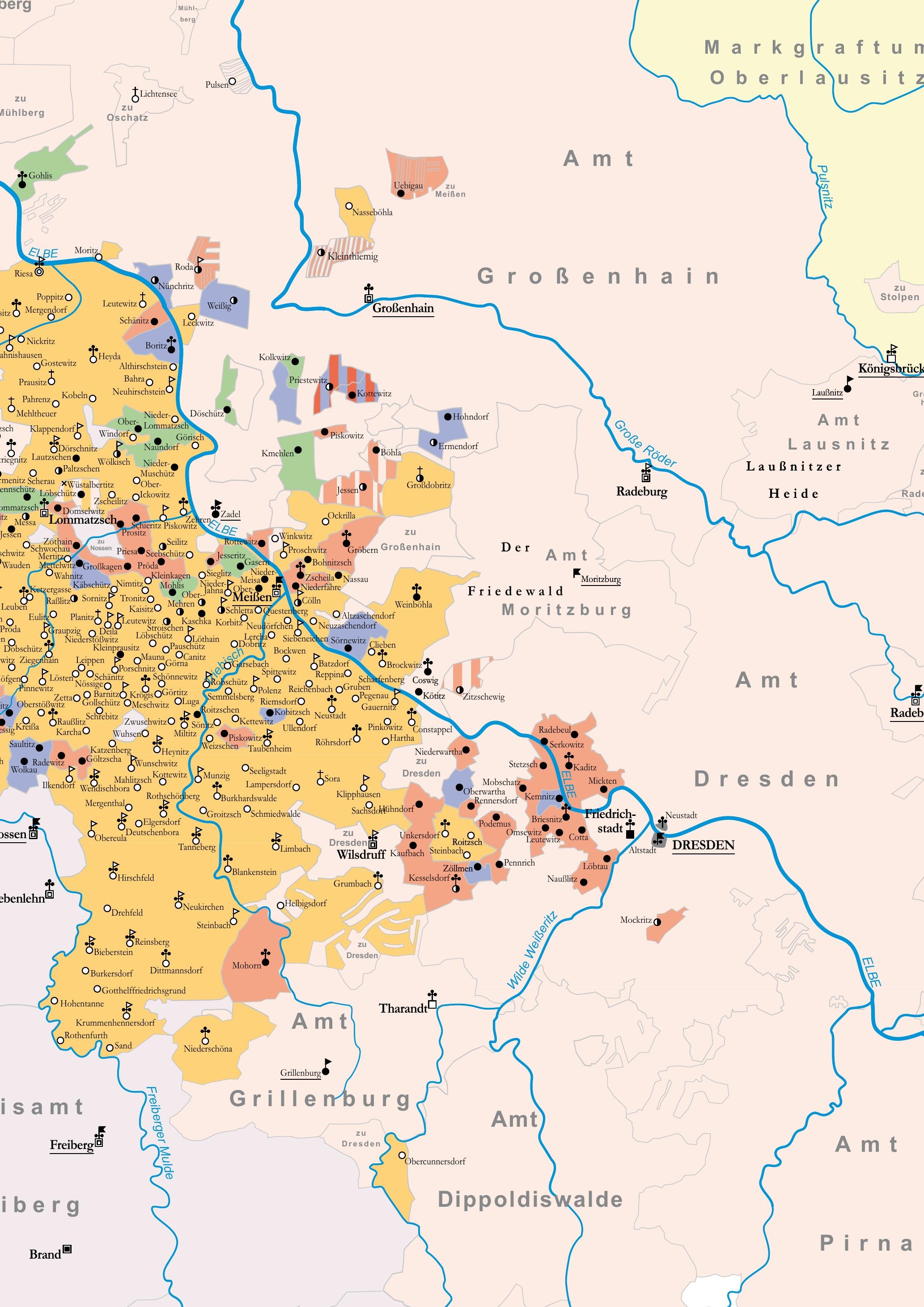


verstärkte. Das nördliche Gebiet um Riesa wurde vom Landkreis Großenhain abgetrennt; es bildete fortan den Kreis Riesa. Die Kreise Meißen, Oschatz und Döbeln blieben bestehen. Die frühere Grenze der Regierungsbezirke wurde nun zur Grenze zwischen den Bezirken Leipzig und Dresden. Das wiederum führte zu einer stärkeren Ausrichtung auf die Bezirksstädte Dresden und Leipzig.

Alle weiteren Entwicklungen nach 1990 vollzogen sich auf der Grundlage der 1952 geschaffenen Kreis- und Bezirksgrenzen. Die Landkreise Riesa und Großenhain wurden 1994 zusammengeschlossen und 2008 mit dem Kreis Meißen vereinigt. Der Landkreis Oschatz ging 1994 im Landkreis Torgau-Oschatz auf, der wiederum 2008 zum Kreis Nordsachsen vergrößert wurde. Kreissitz ist Torgau. Der Kreis Döbeln wiederum ging 2008 im Landkreis Mittelsachsen auf. Kreisstadt ist Freiberg. Die Regierungsbezirke bestanden bis 2008 und wurden dann durch Landesdirektionen ersetzt. Seit 2012 gibt es nur noch eine landeseinheitliche Landesdirektion Sachsen, womit diese Mittelinstanz weitgehend bedeutungslos wurde. Innerhalb der Landkreise Nordsachsen und Mittelsachsen bilden die Dörfer, die der Lommatzscher Pflege zuzurechnen sind, nur marginale Randgebiete. Das sieht im Landkreis Meißen etwas anders aus, da der größte Anteil der Lommatzscher Pflege diesem Kreis zugeordnet ist und hier etwa ein Viertel der Landkreisfläche einnimmt. Die früher eigenständigen Dörfer wurden in zahlreiche Gebietsreformen innerhalb der letzten 70 Jahre zu größeren Einheiten zusammengeschlossen. In der DDR wurden mehrere Gemeindezusammenschlüsse vollzogen. So entstanden in den 1970er Jahren etwa die Gemeinden Planitz-Deila und Jahna-Löthain. Nach dem Ende der DDR wurden die Gemeinden angehalten, größere Strukturen zu bilden. Das führte zu einer ersten Welle von Gemeindezusammenschlüssen. So wurden 1994 die Gemeinden Jahna-Löthain, Planitz-Deila und Krögis mit insgesamt 37 Ortsteilen zur Gemeinde Käbschütztal zusammengefasst. Meist erhielten die Gemeinden den Namen des größten Ortes. In zwei Fällen, Käbschütztal und Ketzerbachtal, wurden neue Gemeindenamen „erfunden“.

Bedingt durch die Vorgaben des Freistaates Sachsen vollzieht sich seit einigen Jahren ein weiterer Konzentrationsprozess. So schlossen sich die Gemeinden Leuben-Schleinitz und Ketzerbachtal zum 1. Januar 2014 der Stadt Nossen an. Zum 1. Januar 2016 wurde die Gemeinde Mochau mit 23 Ortsteilen in die Stadt Döbeln eingegliedert. Auf diese Weise sind riesige kom-

munale Gebilde entstanden. So umfasst die
Stadt Nossen 59 Ortsteile, darunter zahlreiche Dörfer der Lommatzscher Pflege. Die Stadt Lommatzsch ist nicht ganz so stark gewachsen. Ihr gehören inzwischen 39 Ortsteile an. Die Gemeinderäte der Gemeinde Käbschütztal streben eine Auflösung ihrer Gemeinde und eine Angliederung der Ortsteile an die Städte Meißen und Nossen an, was jedoch noch der Zustimmung der beteiligten Kommunen bedarf. Wenn die politischen Vorgaben bestehen bleiben, die auf immer größere Verwaltungseinheiten ausgerichtet sind, dann werden weitere Zusammenschlüsse nicht ausbleiben.

Nach den kommunalen Grenzen des Jahres 2016 liegen im Gebiet der Lommatzscher Pflege nur noch wenige Großgemeinden. Im Landkreis Meißen sind das die Stadt Lommatzsch, die Stadt Nossen (nördlicher Teil des Stadtgebiets), die Gemeinde Käbschütztal, die Gemeinde Diera-Zehren (linkselbischer Teil des Gemeindegebiets), die Gemeinde Hirschstein, die Gemeinde Stauchitz sowie die Stadt Riesa (Teile des Stadtgebiets). Im nördlichen Teil des Landkreises Mittelsachsen liegen die Gemeinden Ostrau und Zschaitz-Ottewig sowie die Stadt Döbeln. Aus dem Landkreis Nordsachsen ist nur die Gemeinde Naundorf mit Verwaltungssitz in Hof unbestritten der Lommatzscher Pflege zuzurechnen.

\section{LEADER-Gebiet Lommatzscher Pflege}

Die Mittel, die die Europäische Union im Rahmen des Europäischen Landwirtschaftsfonds für die Entwicklung des ländlichen Raums (EPLR) ausgibt, werden in Sachsen regional verteilt. Dazu wurden im Freistaat Sachsen LEADER-Gebiete gegründet, die jeweils mehrere Gemeinden umfassen. Das 2007 gebildete LEADER-Gebiet „Lommatzscher Pflege“ umfasste die Stadt Lommatzsch sowie die Gemeinden Diera-Zehren, Hirschstein, Käbschütztal, Klipphausen, Leuben-Schleinitz, Ostrau, Stauchitz, Triebischtal und Zschaitz-Ottewig. So wurde eine Region geschaffen, die über die Landkreisgrenzen hinausreicht. Allerdings gehören ihr auch Gebiete an, die eigentlich nicht zur Lommatzscher Pflege zu rechnen sind, nämlich die Gemeinden Triebischtal (2012 zu Klipphausen) und Klipphausen sowie der rechtselbische Teil der Gemeinde Diera-Zehren. Teile der Lommatzscher Pflege, etwa die Dörfer der früheren Gemeinden Mochau und Ketzerbachtal, gehören nicht der LEADER-Region an. Die Gebietskulisse wurde 2014 um die ländlichen Ortsteile der Stadt Riesa (Böhlen, Canitz, Gostewitz, Jahnishausen, Leutewitz, Mautitz, Nickritz, Oelsitz und Pochra) erweitert.
Herausgeber der „Sächsischen Heimatblätter“ 